\title{
Genetic Algorithm based Optimal Allocation of Distributed Generators for Maximizing the Benefits
}

\author{
Madhuri Hombal ${ }^{1}$, Rekha C $\mathbf{M}^{2}$ \\ M.Tech, IV sem student, Acharya Institute of Technology, Bengaluru ${ }^{1}$ \\ Asst. Professor, Acharya Institute of Technology, Bengaluru ${ }^{2}$
}

\begin{abstract}
This paper proposes a Genetic Algorithm (GA) optimization technique for optimal allocation of Distributed Generators in the distribution network to maximize the benefits to the Local Distribution Company(LDC), as well as the customers connected to the system. The suggested methodology programmed under MATLAB software, helps to identify the optimal buses on which to connect these DG units. The benefits which are considered in this paper are postponement of upgrade investments, reduction of the cost of energy losses and reliability improvement. The implementation of the algorithm is illustrated on a 39-bus test system.
\end{abstract}

Keywords: Distributed Generation, Distribution system, Genetic Algorithm.

\section{INTRODUCTION}

Any plant that is used for generating electricity which is MGs for the Extra High Voltage (EHV) Network. In [4], a connected to the electricity distribution networks is known novel methodology which is load flow based, is aimed at as Distributed Generator. Distributed Generation (DG) quantifying the benefits to a network in terms of refers to power generation at the point of consumption. investment deferral arising from connecting DG units in Generating power on-site, rather than centrally, eliminates the distribution network. In [5], the decisions for optimal the cost, complexity and interdependencies and sizing and siting of DG units are obtained through a costinefficiencies associated with transmission and benefit analysis which is carried out on an hourly basis. distribution. DG implies the modular, small-scale Here a new heuristic approach is proposed. In [6], the generation of power from systems that are often relatively investment deferral produced by the connection of DG small, ranging in size from less than a KW to a few tens of units is investigated on a generic distribution network. MW. DG can be powered by both conventional and Here different DG locations and two different renewable energy sources. With the challenges currently technologies i.e., CHP and wind power, with their facing electricity generation and for the sustainable energy corresponding security contributions, are considered. The supply infrastructure renewable DG becomes the vital authors of papers [1] to [5] only considered dispatchable option, since they are both inexhaustible and non- DG units.

polluting. Optimal location and capacity are the main issue of studies done on the DGs as the proper sizing and The second benefit is to reduce the cost of energy losses. placement of DG allow the system to gain the maximum Towards achieving this second benefit the analytical benefits from the DG, on the other side, improper methods are used to determine the optimal location to placement or sizing of DGs may cause undesirable effects. place a DG in radial as well as networked systems to

There are a number of benefits that can be minimize the power loss of the system as in [7]. Here a achieved from the installation of DG units, among which time-varying load and DG power were considered. In three are considered in this paper.

[8], annual energy losses variations are computed when

The first benefit is to postpone the upgradation different penetration and concentration levels of DG are investments. In [1], a multi-period optimal power flow connected to a distribution network. In [9], analytical was used in order to capture the effects of network expressions for finding optimal size and power factor of 4 investment deferral on DG expansion and to optimally types of DG units. In [10], the authors proposed a multiallocate the DG units in a distribution network. In [2], an objective DG allocation approach to minimize the efficient methodology is proposed for the optimal losses(real and reactive). However, all the works allocation and sizing of switched shunt capacitors in radial presented in the papers from[7] to [10] considered only distribution systems. The methodology proposed here is used to maximize the savings from reduced energy losses and due to costs that are avoided due to investment deferral in the network expansion. In [3], the investment deferral is brought by integrating Microgenerators (MGs) into distribution network. Here, a methodology is proposed to assess investment deferral resulting from conventional type of DG units with dispatchable DG sizes. However in [11], renewable DG units in the distribution system are considered. Here, a methodology has been proposed for optimally allocating different types of renewable DG units in the distribution system so as to minimize the annual energy loss. In [12] also, renewable DG units are considered, wherein, a multiperiod AC 
Optimal Power Flow(OPF) is used to determine the optimal accommodation of renewable DG units in a way that minimizes the system energy losses. In [13], a novel multi-objective planning framework is presented for the integration of stochastic and controllable Distributdd Energy Resources(DERs) in the distribution grid.

In all the above literatures, the main focus was to reduce the total annual energy losses. This is not an accurate representation of LDC requirements, but considering the cost of annual energy losses is the true representation. So unlike the previous literatures that focused on minimizing the cost of the energy losses using fixed energy price,fa methodology to evaluate the cost of energy losses in distribution systems utilizing variable hourly cost of energy is proposed in this paper.

The third benefit is improved reliability of the power supply for different customers. The publications [14] and [15] present some work in the areas of investigating the impacts of DG units on system reliability. In [14], a multi objective formulation for the siting and sizing of DG resources into existing distribution networks is proposed considering the system reliability. A method to determine optimal operating strategy is presented in [15], for DG incorporating reliability worth evaluation of a distribution system.

However, the publications [14] and [15] presented the work considering conventional dispatchable DGs only.

Referring to the above literatures, we can say that a very little work has been addressed the renewable DGs. Therefore, this paper presents a methodology which evaluates the economic benefits of addressing the renewable DGs.

The main factors that are considered in the preseBt methodology are:

1. The uncertainty associated with the renewable DG output

2. Variable hourly cost of energy

The search space of optimal location and capacity of DGs is wide and different optimization methods are used in this field for the sake of power loss minimization, cost reduction, profit maximization and environmental emission reduction.

A Genetic Algorithm (GA) based methodology is employed to solve optimization problem that maximizes the benefit of the system by the optimal placement of DGs.

\section{PROBLEM DESCRIPTION}

\section{A. Cost of System Upgrading $\left(C_{U}\right)$}

This is the cost which includes the investments which are necessary to upgrade any distribution network so as to meet the growing energy demand with the inclusion of new generators or loads connected to the grid.

System upgrade cost is the sum of lines' reinforcement cost and protection and metering equipment upgrade costs.

The wind based DG is modelled by 14 states model and the load is modelled by 8 states model. The procedure for evaluating the cost of system upgrades is described as follows: a) For each state $\mathrm{s}$, go through steps b to d

b) For each year y, go through steps c to d

c) Update loads with annual rise, and run load flow analysis for stat $\mathrm{s}$ and year $\mathrm{y}$

For each line, record the year $y$ in which upgrade is required and calculate the corresponding Net Present Value (NPV) of the cost of upgrade of each line for each state $\mathrm{s}$.

For each line, arrange the combined generation and load states in descending order according to the calculated NPV.

For each line, if the probability of the state corresponding to the maximum cost of upgrade is above the RF, proceed to step g; if not, proceed to the next state. If the sum of the probabilities of this state and the previous state(s) is higher than the RF go to step g; if not, proceed to next state and repeat the previous statement.

Record this upgrade cost and repeat step f for the next line.

The NPV of the required reinforcement investments during the period under study can be evaluated using the following formula:

$$
N P V_{\text {upgrade }}=\sum_{k=1}^{M} N P V_{k}=\sum_{k=1}^{M} \frac{C_{k}}{(1+d)^{i_{k}}}
$$

where $N P V_{k}$ is the NPV of the reinforcement $k ; C_{k}$ is the cost of the reinforcement $k ; d$ is the discount rate; $M$ is the total number of required reinforcements; $i_{k}$ is the year when the reinforcement is required.

\section{Cost of Energy Losses $\left(C_{L}\right)$}

When the DG units are installed in a distribution network, the installation affects the energy losses. The cost of annual energy losses should be calculated hourly because the load keeps varying. So the variable hourly prices of energy is assumed to better assess the effect of renewable DG on system losses.

The cost of annual energy losses is evaluated by

$C_{E_{\text {loss } y}}$
$=\left(\frac{1}{N_{y}}\right) X \sum_{z=1}^{N_{y}}\left(\left[S_{z}\right]_{8760 X N_{S}} X\left[P_{\text {loss }}\right]_{1 X N_{S}}\right)^{T} X C_{8760 X 1}$

Where $S_{z}$ is a binary variable defined as,

$$
S_{z}=[]_{8760 \times N_{s}} \quad \forall z=1,2, \ldots, N_{y}
$$

Where $N_{s}$ is the total number of states of the combined load and generation model; $N_{y}$ is the total number of scenarios.

$$
N P V_{\text {loss }}=\sum_{y=1}^{Y r s} \frac{C_{E_{\text {loss } y}}}{(1+d)^{y}}
$$

\section{Cost of Interruption $\left(\mathrm{C}_{\mathrm{I}}\right)$}

The distribution system is an important link between the transmission-generation system and customers. These links are radial, which makes them susceptible to outage due to failures of a single element. 
The following assumption is made to evaluate the cost of interruption in the distribution networks with renewable DG connections.

Islanding mode of operation is assumed to be allowed, which benefits the customers. Islanded system means, a distribution network is fed from a transmission network, and when the connection to the transmission system is lost, the distribution network is islanded. The DG units can supply the system loads during scheduled or unscheduled outage events, which can improve system reliability.

For an island to be successful, the generated power from all DG units within the island should be higher than or equal to a certain percentage of load required power.

The Genetic Algorithm is used in this paper to find the optimal bus for placing the DG and also to find the optimal size of the DG unit.

\section{GENETIC ALGORITHM AND ITS IMPLEMENTATION}

Genetic Algorithm (GA) is an optimization and search technique based on the principles of genetics and natural selection. GA is population based searching algorithm.

The smallest unit of a GA is called a gene, which represents a unit of information in the problem domain. A series of genes, known as a chromosome, represents one possible solution to the problem. A series of chromosomes is known as population. Each gene in the chromosome represents one component of the solution pattern. The most common form of representing a solution as a chromosome is a string of binary digits. Each bit in this string is a gene. The process of converting the solution from its original form into the bit string is known as coding. The chromosome should in some way contain information about solution which it represents. The most used way of encoding is a binary string.

Generally GA comprises of following steps

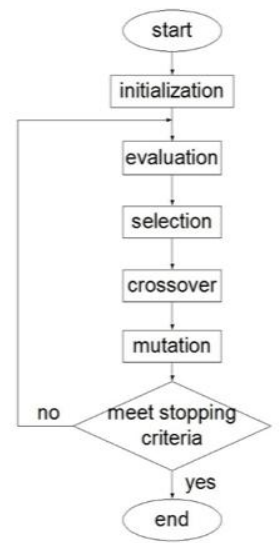

The selection method used in the present paper is Roulette Wheel selection.

\section{Roulette Wheel Selection method:}

Parents are selected according to their fitness. The better the chromosomes are, the more chances to be selected they have. Imagine a wheel where are placed all chromosomes in the population, every has its place big accordingly to its fitness function, like on the following picture.

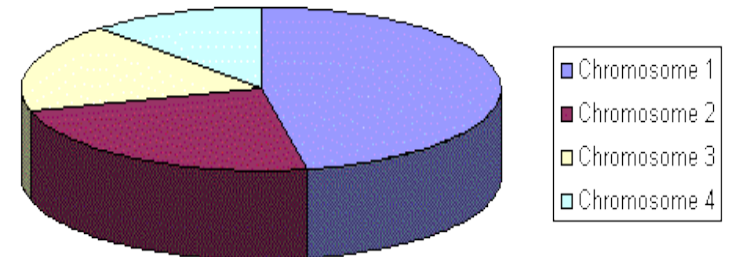

Chromosomes with bigger fitness value will be selected.

Crossover: Crossover selects genes from parent chromosomes and creates a new offspring. Crossover may be regarded as artificial mating in which chromosomes from two individuals are combined to create the chromosome for the next generation. The simplest way how to do this is to choose randomly some crossover point and everything before this point copy from a first parent and then everything after a crossover point copy from the second parent

The idea is that some genes with good characteristics from one chromosome may as a result combine with some good genes in the other chromosome to create a better solution represented by the new chromosome.

$\begin{array}{ll}\text { Chromosome } 1 & 11011 \mid 00100110110 \\ \text { Chromosome 2 } & 11011 \mid 11000011110 \\ \text { Offspring 1 } & 11011 \mid 11000011110 \\ \text { Offspring 2 } & 11011 \mid 00100110110\end{array}$

After a crossover is performed, mutation takes place. Mutation changes randomly the new offspring. For binary encoding we can toggle a few randomly chosen bits from 1 to 0 or vice versa. Mutation can then be following;

Original offspring 1

1101111000011110

Original offspring 2

1101100100110110

Mutated offspring 1

1100111000011110

Mutated offspring 2

In this paper, a GA optimization technique has been used for finding the optimal sizes and locations of DG units. GA is utilized to find the optimal buses suitable for placing the DG.

The coding is done using MATLAB software. A set of chromosomes is created which indicates a set of buses. In this population of chromosomes, one bus which is considered to be the global best is chosen. With this global best bus, local best bus which is generated in each iteration is compared. If the local best variable is good compared to global best, then the local variable will replace the global best variable, otherwise the global best will remain untouched. At the end of all the iterations, the global best variable is nothing but the best bus number suitable to place the DG. 


\section{PROBLEM FORMULATION}

In this paper, the proposed DG planning problem formulation is presented, which is classified as mixed integer nonlinear programming. For combining the effect of DG units' installation on system upgrade, energy losses and reliability, the following objective function is proposed.

Minimize:

Cost $=\operatorname{Cost}(\mathrm{s})$ of Objective $(\mathrm{s})$ $+10^{8} \times \sum_{c}^{n c} x_{c}-$ Incentives (1)

where $\mathrm{x}_{\mathrm{c}}$ is a binary variable corresponding to constraint $\mathrm{c}$ (the second term represents a penalty factor for violating constraint $\mathrm{c})$; $\mathrm{nc}$ is the total number of constraints.

The cost(s) of objective(s) in (1) can be the individual cost or sum of different costs like system upgradation cost, cost of energy losses and cost of interruption.

Subject to

$$
\begin{aligned}
& P_{G_{i_{s y}}}-P_{L_{i_{s y}}}= \sum_{k=1}^{n} V_{i_{s y}} V_{k_{s y}} Y_{i k} \\
& \mathrm{X} \cos \left(\theta_{i k}+\delta_{k_{s y}}-\delta_{i_{s y}}\right) \quad \forall i, s, y \\
& Q_{G_{i_{s y}}}-Q_{L_{i_{s y}}}=-\sum_{k=1}^{n} V_{i_{s y}} V_{k_{s y}} Y_{i k} \\
& \mathrm{X} \sin \left(\theta_{i k}+\delta_{k_{s y}}-\delta_{i_{s y}}\right) \quad \forall i, s, y
\end{aligned}
$$

where $\mathrm{i}$ and $\mathrm{k}$ are the bus number; $\mathrm{n}$ is the total number of buses in the system under study; $\mathrm{s}$ is the state number; $\mathrm{y}$ is the year under study; $\mathrm{P}_{\mathrm{L}}$ and $\mathrm{Q}_{\mathrm{L}}$ are the active and reactive power demands; $\mathrm{P}_{\mathrm{G}}$ and $\mathrm{Q}_{\mathrm{G}}$ are the active and reactive generated powers.

Voltage limits constraints:

$$
\mathrm{V}_{\text {min }} \leq \mathrm{V}_{\text {isy }} \leq \mathrm{V}_{\text {max }} \quad \forall i, s, y
$$

Maximum penetration:

Maximum penetration is taken so as to limit maximum reverse power flow at $60 \%$ of substation rating during minimum load conditions

$$
\sum_{i=1}^{n} P_{D G D_{i}}+P_{D G W_{i}} \leq 0.6 X P_{\text {main }}+0.3 X \sum_{i=1}^{n} P_{L_{i}}
$$

Where PDGD,PDGw and Pmain are the generated power from dispatchable DG units, wind DG units and main substation respectively.

\section{SAMPLE CASE STUDY}

Consider the distribution system which contains a mix of residential, commercial and industrial customers. The system under study is a 39 bus system.

The buses are arbitrarily selected and located as shown in the fig. 2

Four different cases are considered in this paper, which are

1) Base case

2) Dispatchable DG

3) Wind based DG

4) Mix of Dispatchable and wind based DG Copyright to IJARCCE
There are different scenarios in each case. Table I gives the objectives and the Risk Factor(RF) for each

\begin{tabular}{|c|c|c|c|c|}
\hline Case & DG type & Scenario & Objective(s) & $\mathbf{R F}$ \\
\hline $\mathbf{A}$ & No DG & A. 0 & No objectives (Base Case) & \multirow{5}{*}{0} \\
\hline \multirow{4}{*}{ B } & \multirow{4}{*}{ Disp. } & B.1 & UG & \\
\hline & & B. 2 & EL & \\
\hline & & B. 3 & INT & \\
\hline & & B.4 & $\mathrm{UG}+\mathrm{EL}+\mathrm{INT}$ & \\
\hline \multirow{4}{*}{ C } & \multirow{4}{*}{ Wind } & C.1.a & \multirow{2}{*}{ UG } & $3 / 8760$ \\
\hline & & C.1.b & & $6 / 8760$ \\
\hline & & C.2 & EL & \multirow{6}{*}{$3 / 8760$} \\
\hline & & C. 3 & $\mathrm{UG}+\mathrm{EL}$ & \\
\hline \multirow{4}{*}{ D } & \multirow{4}{*}{$\begin{array}{l}\text { Disp. And } \\
\text { Wind }\end{array}$} & D.1 & UG & \\
\hline & & D. 2 & EL & \\
\hline & & D. 3 & INT & \\
\hline & & D. 4 & UG+EL+INT & \\
\hline
\end{tabular}
scenario.

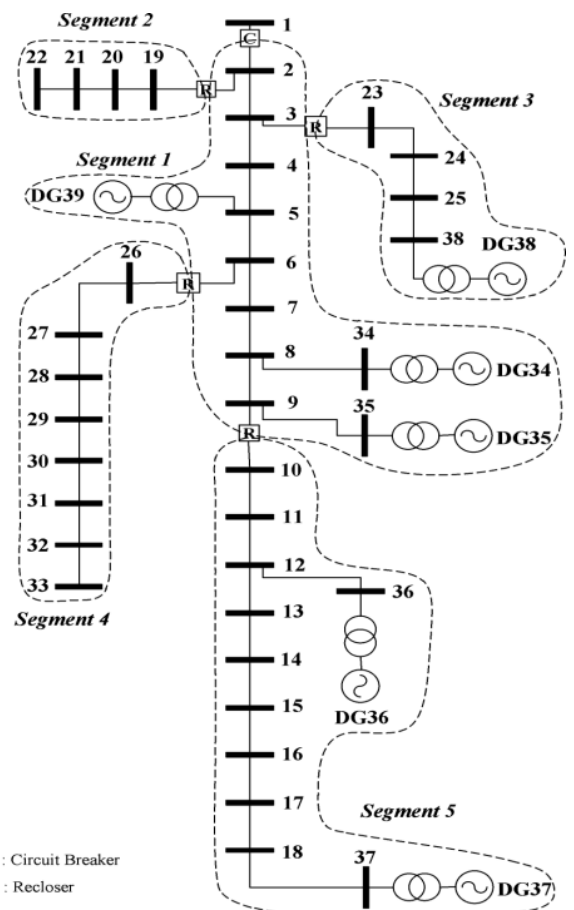

Fig.2 System under study

TABLE I

DIFFERENT SCENARIOS

RESULTS AND DISCUSSIONS

Table II shows the detailed study made for a 20 -year period

Case A. Base Case Results(No DG)

The base case is defined by scenario A.0.

For this case,

$$
\text { Total cost }=\mathrm{UG}+\mathrm{EL}+\mathrm{INT}
$$

$$
2265683=1342301+234,532+688850
$$

i.e., system upgrade cost's share is $59.2 \%$, Energy Losses' share is $10.4 \%$, whereas interruptions share is $30.4 \%$.

These costs are system independent. These costs show that the system upgrade cost has the highest share.

\section{Case B. Dispatchable DG Results}

The Dispatchable DG is denoted by four different scenarios and different objectives as shown in table I.

Scenario B1: The main objective is reduction of Upgradation Cost(UG)

As given in table II,

The cost of UG for scenario B1 $=303,541$ 
The cost of UG for base case(i.e.,for A0)=1,342,301

Therefore, reduction in the cost of upgrades is $77.39 \%$, which is very significant.

This saving in the cost we obtained, because the upgradation of the lines has been deferred or postponed to further years. For ex., required upgradation at $3^{\text {rd }}$ year has been deferred to the $19^{\text {th }}$ year.

Referring to table II,

The cost of EL for scenario B $1=155,590$

The cost of EL for base case=234,532

Therefore, reduction in the cost of EL is $33.7 \%$, which represents a good result, because this is not the objective in this scenario.

The cost of interruption is not affected due to smaller capacities of DG units in segments 3 and 5.

The total cost for scenario B1=UG+EL+INT

$303,541+155,590+688,850=1,147,981$

The total cost for scenario $\mathrm{A} 0=2,265,683$

Therefore, the total saving is $\mathbf{4 9 . 3 \%}$, which proves that the proposed methodology can reduce system cost significantly.

Scenario B2:The main objective is reduction of EL(Energy Loss):

As given in table I,

The cost of EL for scenario B $1=117,840$

The cost of EL for base case(i.e.,for A0) $=234,532$

Therefore, reduction in the cost of Energy Losses is $49.76 \%$

Referring to table II,

The cost of UG for scenario B2 $=736990$

The cost of UG for base case $=1342301$

Therefore, reduction in the cost of UG is $45.1 \%$

Again referring to table II,

The cost of Interruption for scenario B2 is 678,505

The cost of Interruption for base case is 688,850

which shows that there is a slight reduction in the cost of interruption, which is because of the higher capacities of DG units in segments $3 \& 5$ compared to scenario B1. Reducing the cost of interruption is anyway, not the main objective of this scenario.

The total cost for scenario $\mathrm{B} 2=\mathrm{UG}+\mathrm{EL}+\mathrm{INT}$

$736,990+117,840+678,505$

The total cost for scenario $\mathrm{A} 0=2,265,683$

Therefore, the total \% saving for scenario B2 is $\mathbf{3 2 . 3 \%}$

Comment: Comparing the results of scenario B1 and B2 we can conclude that the system upgrade cost is more effective, since the total saving in scenario B1 is better than in B2.

Scenario B3: The main objective is reduction of cost of interruption (INT)

As given in table II,

The cost of INT for scenario B3 $=563,443$

The cost of INT for base case $=688,850$

Therefore, reduction in the cost INT is $18.2 \%$

Referring to table II,

The cost of upgrade for scenario B3 $=1,149,353$

The cost of upgrade for base case $=1,342,301$

Therefore, the \%saving is $14.4 \%$

Again referring to table II,

The cost of EL for scenario B3 $=168,163$
The cost of EL for base case $=234,532$

Therefore, the \%saving is $28.3 \%$

Therefore, the total cost for scenario B3=UG+EL+INT

$1,149,353+168,163+563,443=1,880,958$

The total cost for scenario $\mathrm{A} 0=2,265,683$

Therefore, the total \% saving for scenario B3 is $\mathbf{1 6 . 9 8 \% . ~}$

Comment: The above result shows that the cost of interruption is the least effective cost in the case under study.

Scenario B4: The main objective is reduction of the three considered costs i.e., UG+EL+INT

As can be seen from the table II, the results are same as scenario B1

Conclusion: For the case under study, the upgrades cost is the most effective cost and the Interruption cost is the least effective cost

\section{Case C: Wind Based DG Results}

Since in this case, only wind based DG units are considered, the cost of interruption is not affected. For evaluating the cost of lines' upgrades in case of wind based DG units, if the Risk Factor(RF) is taken to be zero, the cost is greater than or equal to the cost of lines' upgrade without DG.

The scenarios under this case are characterized by nonzero RF. The risk of overloading arises from neglecting one or more states that contribute the most in the reinforcement requirements. The different scenarios under this case are $\mathrm{C} 1 \mathrm{a}, \mathrm{C} 1 \mathrm{~b}, \mathrm{C} 2, \mathrm{C} 3$. For the study of this case, $\mathrm{RF}$ is taken with different values for different scenarios as shown in the table II.

Scenario C1a: The main objective is reduction of Upgradation Cost(UG)

The RF value is taken as 3 hrs./year. The results of the allocation problem show a savings of $8.49 \%$ for upgrade costs and $13.1 \%$ for the cost of energy losses

Scenario C1b: The main objective is reduction of Upgradation Cost(UG)

The RF value is taken as 6hrs/year. The results of the allocation problem show a savings of $27.11 \%$ for the upgrade costs and $20.6 \%$ for the cost of energy losses.

Comment: Comparing scenarios $\mathrm{C} 1 \mathrm{a}$ and $\mathrm{C} 1 \mathrm{~b}$, we can conclude that as the RF increases, the cost of upgrading keeps decreasing. i.e., as the risk of overloading the lines is increased, the expected upgrade costs keep decreasing as shown in the fig. 5. The curve shows that the reduction in the UG costs is not uniform. This is because, the costs depend upon the wind regime, load curve and system under study.

Scenario C2: The main objective is reduction of Cost of Energy Losses

$\mathrm{RF}$ taken in this scenario is $3 \mathrm{hrs}$./year. Table II shows that cost of EL is reduced by $34.7 \%$ but the cost of UG is higher than the base case by $36.6 \%$. But, reducing the UG cost is not the objective of this scenario.

Conclusion: Comparing scenarios $\mathrm{C} 2$ and B2, where the main objective of both is the reduce the cost of EL, the reduction in EL in scenario $\mathrm{C} 2(34.7 \%)$ is smaller compared to the scenario B2(49.76\%). Hence we can conclude that this result is due to the variability and uncertainty of wind based DGs. 
Scenario C3: The main objective is reduction of Cost of Energy Losses and Upgradation costs i.e. UG+EL RF taken in this scenario is 3 hrs./year. The total savings in the costs of EL and UG is $6.48 \%$. This value is slightly greater than scenario $\mathrm{C} 1 \mathrm{a}$.

Conclusion: When different scenarios in case $\mathrm{C}$ are compared, the results show that for wind based DG units, the most effective cost is the cost of energy losses. The ability of reducing the cost of upgrades and energy losses is limited in case of wind based DG units, because of their stochastic nature.

\section{Case D:Wind and Dispatchable DG Results}

Different scenarios under this case are D1,D2,D3 and D4 In this case, along with the dispatchable DGs, $40 \%$ of wind based DGs are assumed to be installed.

i.e., the capacities of the wind DG units $\geq$ two-thirds the capacities of the dispatchable DG units.

So the constraint should be,

$\sum_{i=1}^{n} P_{D G W_{i}} \geq \frac{2}{3} \times \sum_{i=1}^{n} P_{D G D_{i}}$

Scenario D1: The main objective is reduction of Upgradation Cost(UG)

Referring to the table II and comparing the savings in the cost of upgrades in scenario D1 and scenario B1(the objective of both are the same), we can say that the savings in the cost of upgrades in $\mathrm{D} 1(70.7 \%)$ is lower than the savings in the cost of upgrades in B1(77.39\%). The reason for this is the green energy constraint (A).

As given in the table II, the capacity of the wind DG units is $0.6 \mathrm{MW}$, which is exactly $\frac{2}{3}$ of the total capacity

of the dispatchable DG units as given in the equation (A).

Scenario D2: The main objective is reduction of Energy Losses(EL)

Comparing the savings in EL in scenario D2 with the savings in scenario B2, we can say that the savings in $\mathrm{D} 2(49.38 \%)$ is very close to savings in $\mathrm{B} 2(49.76 \%)$. The only difference in this scenario is that this scenario comes with the higher DG capacities(both renewable and dispatchable) as compared to the DG capacities of scenario B2.

Scenario D3: The main objective is reduction of Interruption (INT) costs

Referring to the table II and comparing the results of Scenarios B3 and D3(the objectives of both are same), we can conclude that the maximum savings in the interruption cost in $\mathrm{D} 3(12.97 \%)$ is smaller than the savings in the B3(18.21\%) though the total DG capacity in D3 is higher than the total capacity in B3. The reason for this is the intermittent or stochastic effect of the wind based DGs which limit their contribution in reducing the interruption cost.

Scenario D4: The main objective is reduction of the three considered costs i.e., UG+EL+INT

Results of this scenario are found to be the same as scenario D1.

Comment:Referring to the table II and comparing D4 with B4(the objectives of both are the same), we can conclude that the total savings in D4(46\%) is less than the total savings in B4(49.3\%) but the total DG capacity in
D4 which uses a mix of dispatchable and wind based DG units is more than the total DG capacity of B4 which uses only dispatchable DG units. In scenario B4, RF is 0 while in D4, risk of overloading is 3hrs./year.

\section{CONCLUSION}

In this paper, a GA based optimization approach is proposed to optimally allocate different types of DG units into the distribution system. The main aim of this paper is to consider the renewable DG units and their uncertainty and variability by defining a factor called Risk Factor(RF), which represents the expected duration of overloading the lines per year. This paper mainly concentrates on optimal allocation of different types of DGs in the distribution system, to maximize the benefits through the reduction of losses(energy and interruption) and by deferring or postponing the investment upgrades. The cost of interruption is calculated by considering the nonlinear, time dependent $\mathrm{CDF}$, which gives more accurate results, as the effect of renewable DGs is mostly vary according to the time during islanded mode of the distribution network.

Moreover, the proposed methodology in the paper mainly considers the hourly cost of energy for calculating the Net Present Value(NPV) of the energy losses.

Using GA based optimization approach, the best locations for placing the different types of DG units are identified and the proposed methodology can be easily applied to any type of radial distribution system. Moreover, the proposed methodology can be applied to any type of DG units such as dispatchable or renewable DG units or in any combination of these DG units.

\section{REFERENCES}

[1] A. Piccolo and P. Siano, "Evaluating the impact of network investment deferral on distributed generation expansion," IEEE Trans. Powe Syst., vol. 24, no. 3, pp. 1559-1567, Aug. 2009.

[2] H.M. Khodr, F.G.Olsina, P. M. De Oliveira-De Jesus, and J. M.Yusta, "Maximum savings approach for location and sizing of capacitors in distribution systems," Elect. Power Syst. Res., Elsevier, vol. 78, no. 7, pp. 1192-1203, Jul. 2008

[3] G. Pudaruth and F. Li, "Costs and benefits assessment considering deferral of assets expansion in distribution systems," in Proc. 42nd Int.Universities Power Eng. Conf., UPEC 2007, 2007, pp. 872-878.

[4] Y. Zhang, C.Gu, and F. Li, "Evaluation of investment deferral resulting from microgeneration for EHV distribution networks," in Proc. IEEEPower and Energy Society General Meeting, 2010, pp. 1-5.

[5] W. El-Khattam, K. Bhattacharya, Y. Hegazy, and M. M. A Salama, "Optimal investment planning for distributed generation in a competitive electricity market," IEEE Trans. Power Syst., vol. 19, no. 3, pp. 1674-1684, Aug. 2004.

[6] D. T. C. Wang, L. F. Ochoa, and G. P. Harrison, "DG impact on investment deferral: Network planning and security of supply," in Proc.IEEE Power and Energy Society General Meeting, 2010, p. 1.

[7] C. Wang andM. H. Nehrir, "Analytical approaches for optimal placementof distributed generation sources in power systems," IEEE Trans.Power Syst., vol. 19, no. 4, pp. 2068-2076, Nov. 2004.

[8] V. H. M. Quezada, J. R. Abbad, and T. G. S. Roman, "Assessment of energy distribution losses for increasing penetration of distributed generation," IEEE Trans. Power Syst., vol. 21, no. 2, pp. 533 540,May 2006.

[9] D. Q. Hung, N. Mithulananthan, and R. Bansal, "Analytical expressions for DG allocation in primary distribution networks," IEEE Trans.Energy Convers., vol. 25, no. 3, pp. 814-820, Sep. 2010. 
[10] L. F. Ochoa, A. Padilha-Feltrin, and G. P. Harrison, "Evaluating distributed generation impacts with a multiobjective index," IEEE Trans.Power Del., vol. 21, no. 3, pp. 1452-1458, Jul. 2006.

[11] Y. M. Atwa, E. F. El-Saadany, M. M. A. Salama, and R. Seethapathy, "Optimal renewable resources mix for distribution system energy loss minimization," IEEE Trans. Power Syst., vol. 25, no. 1, pp. 360-370, Feb. 2010.

[12] L. F. Ochoa and G. P. Harrison, "Minimizing energy losses: Optimal accommodation and smart operation of renewable distributed generation," IEEE Trans. Power Syst., vol. 26, no. 1, pp. 198-205, Feb. 2011

[13] A. Alarcon-Rodriguez, E. Haesen, G. Ault, and J. Dries, "Multiobjective planning framework for stochastic and controllable distributed energy resources," IET Renew. Power Gen., vol. 3, no. 2, pp. 227-238, Jun. 2009.

[14] G. Celli, E. Ghiani, S. Mocci, and F. Pilo, "A multiobjective evolutionary algorithm for the sizing and siting of distributed generation," IEEE Trans. Power Syst., vol. 20, no. 2, pp. 750-757, May 2005.

[15] I.-S. Bae, J.-O. Kim, J.-C. Kim, and C. Singh, "Optimal operatingstrategy for distributed generation considering hourly reliability worth," IEEE Trans. Power Syst., vol. 19, no. 1, pp. 287 292, Feb. 2004.

[16] Y. M. Atwa and E. F. El-Saadany, "Reliability evaluation for distribution system with renewable distributed generation during islanded mode of operation," IEEE Trans. Power Syst., vol. 24, no. 2, pp. 572-581, May 2009.

[17] S. H. Jangamshetti and V. G. Rau, "Site matching of wind turbine generators: A case study," IEEE Trans. Energy Convers., vol. 14, no. 4, pp. 1537-1543, Dec. 1999.

[18] Y. Hegazy, M. Salama, and A. Chikhani, "Adequacy assessment of distributed generation systems using Monte Carlo simulation," IEEE Trans. Power Syst., vol. 18, no. 1, pp. 48-52, Feb. 2003.

[19] C. Chiang, "Genetic-based algorithm for power economic load dispatch," IET Gen., Transm., Distrib., vol. 1, no. 2, pp. 261-269, Mar. 007.

[20] D. Singh and R.Misra, "Effect of load models in distributed generation planning," IEEE Trans. Power Syst., vol. 22, no. 4, pp. 2204-2212, Nov. 2007. 


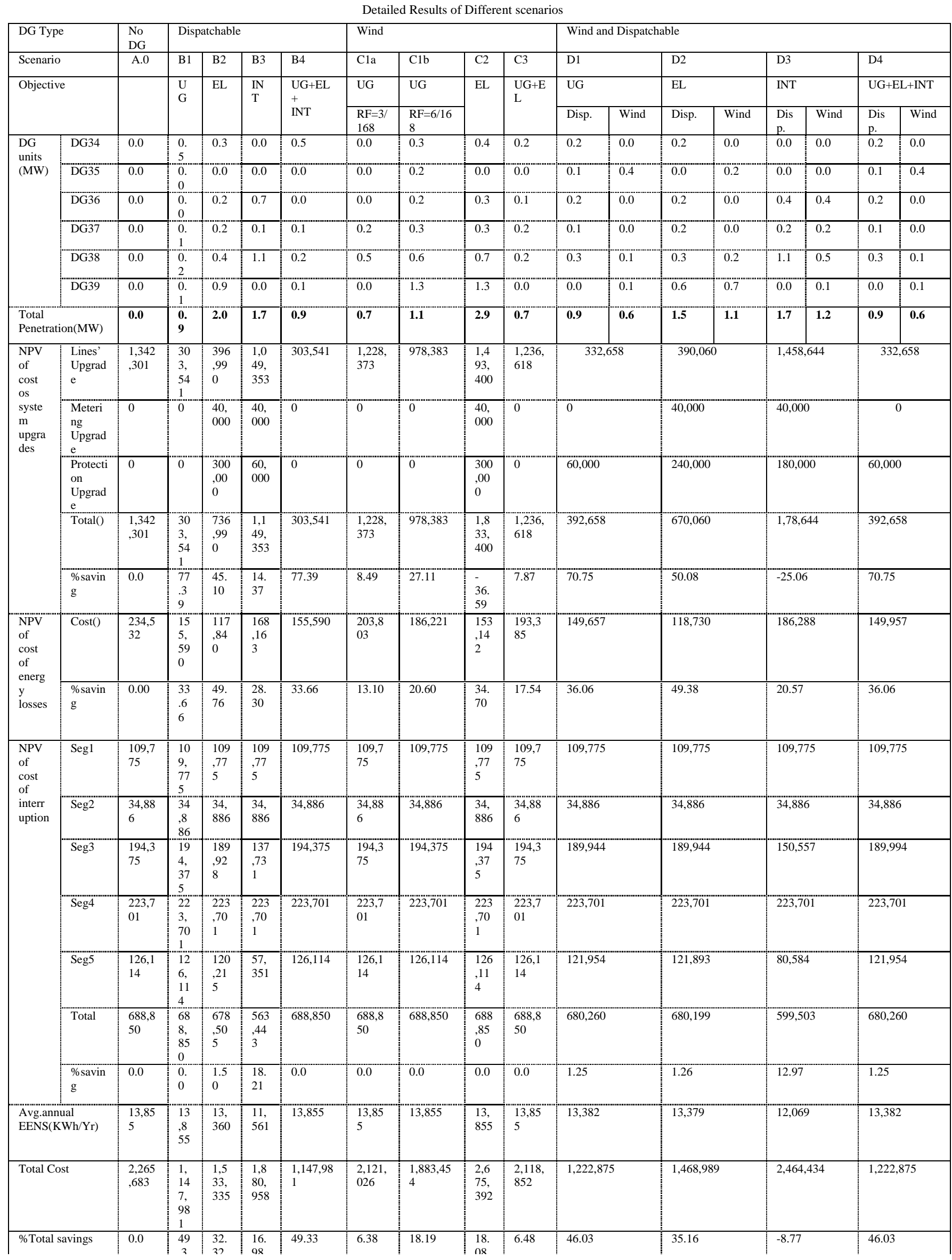

